\title{
Glass-liquid and glass-gel transitions of soft-shell particles
}

\author{
Lara Frenzel, ${ }^{1,2}$ Michael Dartsch, ${ }^{1,2}$ Gerard Martí Balaguer, ${ }^{3}$ \\ Fabian Westermeier, ${ }^{1}$ Gerhard Grübel, ${ }^{1,2}$ and Felix Lehmkühler ${ }^{1,2, *}$ \\ ${ }^{1}$ Deutsches Elektronen-Synchrotron DESY, \\ Notkestr. 85, 22607 Hamburg, Germany \\ ${ }^{2}$ The Hamburg Centre for Ultrafast Imaging, \\ Luruper Chaussee 149, 22761 Hamburg, Germany \\ ${ }^{3}$ Autonomous University of Barcelona, 08193 Bellaterra, Catalonia, Spain
}

(Dated: June 23, 2021) 
We study the structure and dynamics of colloidal particles with a spherical hard core and a thermo-responsive soft shell over the whole phase diagram by means of small-angle X-ray scattering and X-ray photon correlation spectroscopy. By changing the effective volume fraction by temperature and particle concentration, liquid, repulsive glass and attractive gel phases are observed. The dynamics slow down with increasing volume fraction in the liquid phase and reflect a Vogel-Fulcher-Tamann behaviour known for fragile glass formers. We find a liquid-glass transition above 50 vol.\% that is independent from the particles' concentration and temperature. In an overpacked state at effective volume fractions above 1 , the dispersion does not show a liquid phase but undergoes a gel-glass transition at an effective volume fraction of $34 \mathrm{vol.} \%$. At the same concentration, extrema of subdiffusive dynamics are found in the liquid phase at lower weight fractions. We interpret this as dynamic precursors of the glass-gel transition.

Upon supercooling the structural relaxation time of many liquids grows by orders of magnitude before they undergo a glass transition $[1,2]$. In recent years, similar observations have been made for colloidal systems where the volume fraction of the particles defines the sample phase [3]. Due to its importance as a model system for theory and experiments, special attention has been paid to the hard-sphere glass transition [3-5]. With increasing volume fraction, structural order develops in hard sphere systems in the liquid state $[6,7]$. Upon supercooling, i.e., above volume fractions of $\phi \approx 0.5$, the structural correlation time grows and diverges at the glass transition around $\phi_{0} \approx 0.59$. This is described by a VogelFulcher-Tamann (VFT) behaviour similar to other glass formers [1-3], where the relaxation time $\tau$ follows an exponential that diverges at a VFT volume fraction $\phi_{\mathrm{VFT}} \geq \phi_{0}$

$$
\tau \propto \exp \left(\frac{m \phi_{0}}{\phi_{0}-\phi}\right) \text {. }
$$

The so-called fragility index $m$ is a measure of the deviation from an Arrhenius-type behaviour of the viscosity or relaxation time while approaching the glass transition.

Recently, research has been extended to so-called soft colloidal systems that show a much richer phase behaviour, such as different crystalline states observed in dispersions of charged particles [8]. A special class of frequently studied soft particles are microgels or nanogels made from Poly(N-isopropylacrylamide) (PNIPAm). Such PNIPAm particles dispersed in

\footnotetext{
* Corresponding author: felix.lehmkuehler@desy.de
} 
water show a reversible volume phase transition at a lower critical solution temperature (LCST) of $306 \mathrm{~K}$ [9-13]. Below this LCST, the polymer is hydrophilic and the particles hence swollen with water. The interaction is typically described by a repulsive Hertzian potential $[14,15]$. They become hydrophobic above this temperature, releasing the water and forming a collapsed state. Therefore, besides the volume fraction the phase of such a microgel dispersion depends on the temperature $T$ as well. Above the LCST, the particles become attractive, promoting the formation of a colloidal gel at high volume fractions [16$27]$.

As the degree of swelling and thus the direct particle interaction varies with $T$, comparing PNIPAm systems at different temperatures is difficult, in particular using the temperature to change the volume fraction of the dispersion. Therefore, the glass transition of such dispersions has been studied typically at constant temperature, changing the volume fraction only $[11,12]$. Several similarities between the glass transition of hard and soft spheres have been reported [28], however, many properties remain unclear or controversial, such as the fragility, the role of softness, and the relation to gelation and jamming transitions [28-44].

In this work we address the relation between the glass, gel and liquid phases by studying the structure and dynamics of colloidal soft spheres made from a hard spherical $\mathrm{SiO}_{2}$ core and a soft PNIPAm shell by means of small-angle X-ray scattering (SAXS) and X-ray photon correlation spectroscopy (XPCS). By modifying the particle number density via the weight fraction and the particles radius by temperature changes between $288 \mathrm{~K}$ and $318 \mathrm{~K}$ effective volume fractions between $\zeta=0.03$ and $\zeta=1.6$ are reached, covering liquid, glass and gel phases. In the liquid phase, the sample dynamics slows down with increasing volume fraction following a Vogel-Fulcher-Tamann law. At $\zeta_{0}=0.51$, the dynamics falls out-ofequilibrium indicating a liquid-glass transition, independent of the particles' weight fraction and temperature, and thus the interaction potential. At high weight fractions, overpacked states are reached at $288 \mathrm{~K}$, i.e. $\zeta>1$. In this case the system does not show a liquid phase and undergoes a gel-glass transition at $\zeta_{\mathrm{gg}}=0.34$. In the liquid phase at lower weight fractions extrema of subdiffusive dynamics are found at $\zeta_{\text {gg }}$ suggesting the existence of dynamic precursors of the glass-gel transition.

We study the structure and dynamics at weight fractions between $c=4$ wt.\% and 15.8 wt.\% of silica-PNIPAm core-shell nanoparticles. Their hydrodynamic radius is $228 \mathrm{~nm}$ in the swollen state at $15^{\circ} \mathrm{C}$ and $100 \mathrm{~nm}$ in the collapsed state at $45^{\circ} \mathrm{C}$. Details of the synthesis 
of the nanoparticles and details of the XPCS experiment performed at beamline P10 at PETRA III (DESY, Hamburg, Germany) are given in the Supplementary Materials.

In dynamic light scattering (DLS) and XPCS experiments the dynamics of a sample are probed by auto-correlating the intensity $I(q, t)$ at time $t$ and the modulus of the wave vector transfer $q \equiv|\mathbf{q}|=\frac{4 \pi n}{\lambda} \sin (\theta / 2)$. Here, $n$ is the index of refraction which is $\approx 1$ for X-rays, $\lambda$ the wave length and $\theta$ the scattering angle. The correlation function is given by

$$
g_{2}(q, \Delta t)=\frac{\langle I(q, t) I(q, t+\Delta t)\rangle_{t}}{\langle I(q, t)\rangle_{t}^{2}} .
$$

For the samples studied here this $g_{2}$-function can be expressed by the intermediate scattering function $f(q, \Delta t)$ that is given by a Kohlrausch-Williams-Watts (KWW) expression

$$
g_{2}(q, \Delta t)=1+\beta|f(q, \Delta t)|^{2}=1+\beta \exp \left(-2(\Gamma \Delta t)^{\gamma}\right) .
$$

The speckle contrast $\beta$ is defined by coherence properties of the X-ray beam and setup parameters. It is close to 1 for DLS and was found to be 0.18 in the XPCS experiment.

The relaxation time $\tau$ is given by the inverse of the relaxation rate $\tau=\Gamma^{-1}$. The $q$ dependence of $\Gamma \propto q^{p}$ and the KWW-exponent $\gamma$ typically define the type of dynamics, e.g., for diffusion of colloidal particles one obtains $\gamma=1$ and $\Gamma(q)=D_{0} q^{2}$, with the StokesEinstein diffusion coefficient $D_{0}=\frac{k_{\mathrm{B}} T}{6 \pi \eta r}$. This is used in DLS experiments to obtain the particles' hydrodynamic radius $r$ with Boltzmann's constant $k_{\mathrm{B}}$, the known temperature $T$ and solvent viscosity $\eta$.

As the PNIPAm particles deform and deswell at large volume fractions, an effective volume fraction is conventionally used defined by $\zeta=N \cdot V(T)$, with $N$ the particle number concentration and $V(T)$ the volume fraction of a particle in a dilute dispersion at temperature $T$ as obtained by dynamic light scattering $[39,45,46]$. Values around and above $\zeta=1$ are possible with deformed particles and interpenetration of the PNIPAm shell [12, 13, 45, 4750]. Depending on the softness of pure PNIPAm particles the real volume fraction matches $\zeta$ up to approximately $\zeta=0.8[46,51]$. At higher volume fractions the particles are not in swelling equilibrium [31].

Intermediate scattering functions and fits of Eq. 3 to the data are exemplary shown for $c=9$ wt. $\%$ at $T=288 \mathrm{~K}$ and $T=313 \mathrm{~K}$ in the Supplementary Material together with the temperature dependence of the so-obtained $\tau$ and the two exponents $p$ and $\gamma$. Here, we discuss the results as a function of the effective volume fraction of all samples 
studied. Since the radius of the core-shell particles shrinks already below the LCST, each experiment at a fixed weight fraction and temperature corresponds to a different effective volume fraction $\zeta$. The relaxation time $\tau$ is shown in Fig. 1 (a) as a function of $\zeta$. Despite of the different number densities and temperatures, the measured relaxation times $\tau$ seem to collapse on a common master curve. This is surprising because the interaction potential significantly changes with particle swelling and thus with temperature [15]. This may lead to different dynamics at different temperatures, especially below the LCST. Even above 311 $\mathrm{K}$ (open diamonds), where we recently reported gelation due to the appearance of attractive interaction between the particles [26], the data follows the trend for $c<10 \mathrm{wt} . \%$. Samples at higher concentrations do not speed up as discussed in the Supplementary Material and eventually reach a gel phase.

In general, the slowing down with increasing $\zeta$ appears to resemble the behaviour of fragile glass formers reaching the glass transition $[28,52]$. The relaxation time grows steeply up to $\zeta \approx 0.5$ and changes to a slower, linear growth above. We modelled the increase of $\tau$ for $\zeta \lesssim 0.5$ by a Vogel-Fulcher-Tamann (VFT) law

$$
\tau(\zeta)=\tau_{0} \exp \left(\frac{m \zeta_{\mathrm{VFT}}}{\zeta_{\mathrm{VFT}}-\zeta}\right)
$$

where $\tau_{0}$ is a scaling parameter, $m$ the fragility index, and $\zeta_{\mathrm{VFT}}$ is the VFT volume fraction where $\tau \rightarrow \infty$. We fitted Eq. 4 to our data for $0.0036 \mathrm{~nm}^{-1} \leq q \leq 0.017 \mathrm{~nm}^{-1}$ and $c \leq 9$ wt.\% and obtained $\zeta_{\mathrm{VFT}}=0.84 \pm 0.03$ and an index of $m=6.1 \pm 0.2$. In literature, using viscosity measurements broad range of $\zeta_{\mathrm{VFT}}$ has been reported for different soft particle systems ranging from 0.5 to more than 1.2 , mainly depending on the degree of softness $[39,53,54]$. Note that $\zeta_{\mathrm{VFT}}$ found here matches results from pure PNIPAm microgels obtained by DLS [28].

In literature, different definitions of the fragility index are discussed. For instance, the kinetic fragility $m^{*}$ defined as $[29,39,44]$

$$
m^{*}=\left[\frac{\partial \log (\tau)}{\partial\left(\zeta / \zeta_{g}\right)}\right]_{\zeta=\zeta_{g}},
$$

uses a glass transition volume fraction $\zeta_{g}$. Following the definition from Mattson et al. [29] we may fix the glass transition at $\tau / \tau_{0}=10^{5}$ and obtain $\zeta_{g}=0.587$. This is close to the glass transition found for hard spheres [3,39]. At this value we have $m^{*}\left(\zeta_{g}\right)=1.2$. A different prominent choice of $\zeta_{g}$ in Eq. 5 is given by $\zeta_{0}=0.51$. Above $\zeta_{0}$ the VFT fails to describe 


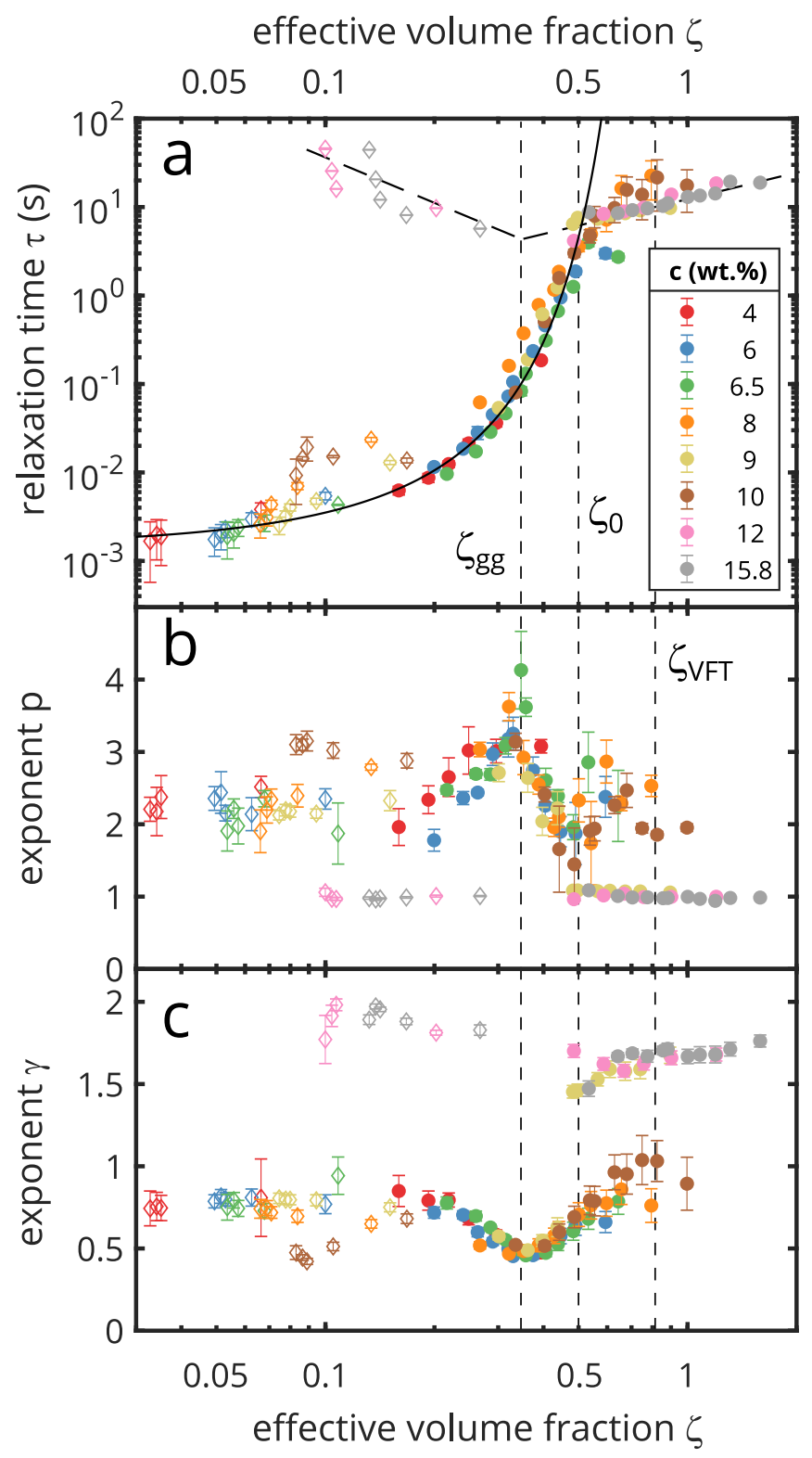

FIG. 1. a) Relaxation time $\tau$ at $q=0.0116 \mathrm{~nm}^{-1}$ as function of volume fraction. Open diamonds correspond to $T \geq 311 \mathrm{~K}$, full circles represent $T<311 \mathrm{~K}$. Solid black line is the fit of the VFT model, the empiric dashed line is a linear fit for high $\zeta$ and a power law for low $\zeta$. The crossover of solid and dashed line is found at $\zeta_{0}=0.510$. b) Exponent $p$ and c) exponent $\gamma$ as function of volume fraction. Vertical dashed lines mark $\zeta_{\mathrm{gg}}, \zeta_{0}$, and $\zeta_{\mathrm{VFT}}$.

the data and we obtain $m^{*}\left(\zeta_{0}\right)=0.93$. Both kinetic fragilities indicate that our core-shell sample is a strong glass former, supporting findings from previous studies on soft particles $[29,39,44]$.

The behaviour for $\zeta>0.5$ and in general for $c \geq 12$ wt.\% shown as dashed line in 
Fig. 1 (a) was empirically modelled by the sum of a power law $\tau \propto \zeta^{-1.7}$ dominating at low volume fractions and a linear relation at high volume fractions. Such a power law behaviour has been reported for PNIPAm-based gels [20,31]. The change from the power law to the linear behaviour is found at $\zeta_{\mathrm{gg}}=0.34$, the crossing of the linear fit and the VFT model is located at $\zeta_{0}=0.510$. In Fig. 1 (b) and (c) we show exponents $p$ and $\gamma$. For $\zeta<\zeta_{0}$ and $c<10$ wt.\% we find $p \geq 2$ and $\gamma \leq 0.8$, which is typical for the liquid state [23, 26]. We find a maximum of $p\left(p_{\max } \approx 4\right)$ that coincides with a minimum of the KWW exponent $\left(\gamma_{\min } \approx 0.6\right)$. Remarkably, both extrema are located at $\zeta_{\mathrm{gg}}$ obtained from the dynamics of the sample at high weight fractions. This may suggest the existence of a dynamic precursor of the glass-gel transition at the same $\zeta$ but lower weight fractions.

Above $\zeta_{0}$, the exponents agree to values obtained at lower volume fractions, i.e. $p=2$ to 3 and $\gamma \approx 0.8$. This suggests that the sample is in a supercooled, liquid state with diffusive dynamics. Notably, the dynamics show similar behaviour reported from light scattering experiments on PNIPAm systems where the temperature was fixed and the volume fraction was changed by modification of the number density of PNIPAm particles [44]. For high weight fractions the exponents describe glassy states above $\zeta_{0}$ with ballistic dynamics indicated by $p \approx 1$ and $\gamma>1$.5. Similar to the relaxation time $\tau$, both exponents of the two highest weight fractions only show weak modulations. In particular, $p \approx 1$ is found for all concentrations, while $\gamma$ follows the same trend found for $\tau$. This implies that these samples do not show a liquid state and stay in a glass or gel phase over the whole range of volume fractions studied.

The sample dynamics will now be compared to structural properties. Therefore, the structure factors $S(q, c, T)$ are calculated via $S(q, c, T)=I(q, c, T) / P(q, T)$, with the single particle form factors $P(q, T)$ measured from a dilute dispersion. The structure factors are shown in Fig. 2 (a)-(d) for four weight fractions. In general, all $S(q)$ show the same tendencies with changing temperatures. At low temperature, the first peak is found around $q=0.02$ to $0.03 \mathrm{~nm}^{-1}$ where its position $q_{\max }$, reflecting the effective next-neighbour distance, and its height $S_{\max }=S\left(q_{\max }\right)$ depend on the weight fraction. In addition, for $q \rightarrow 0$ the structure factors converge around $S(q \rightarrow 0) \approx 0.5$ to 0.6 .

With increasing temperature, the peak position shifts to larger $q$ and the peak height decreases, indicating a reduced particles size and thus a reduced volume fraction and lessordered next-neighbour shell. This is accompanied by a gradual increase of the contribution 

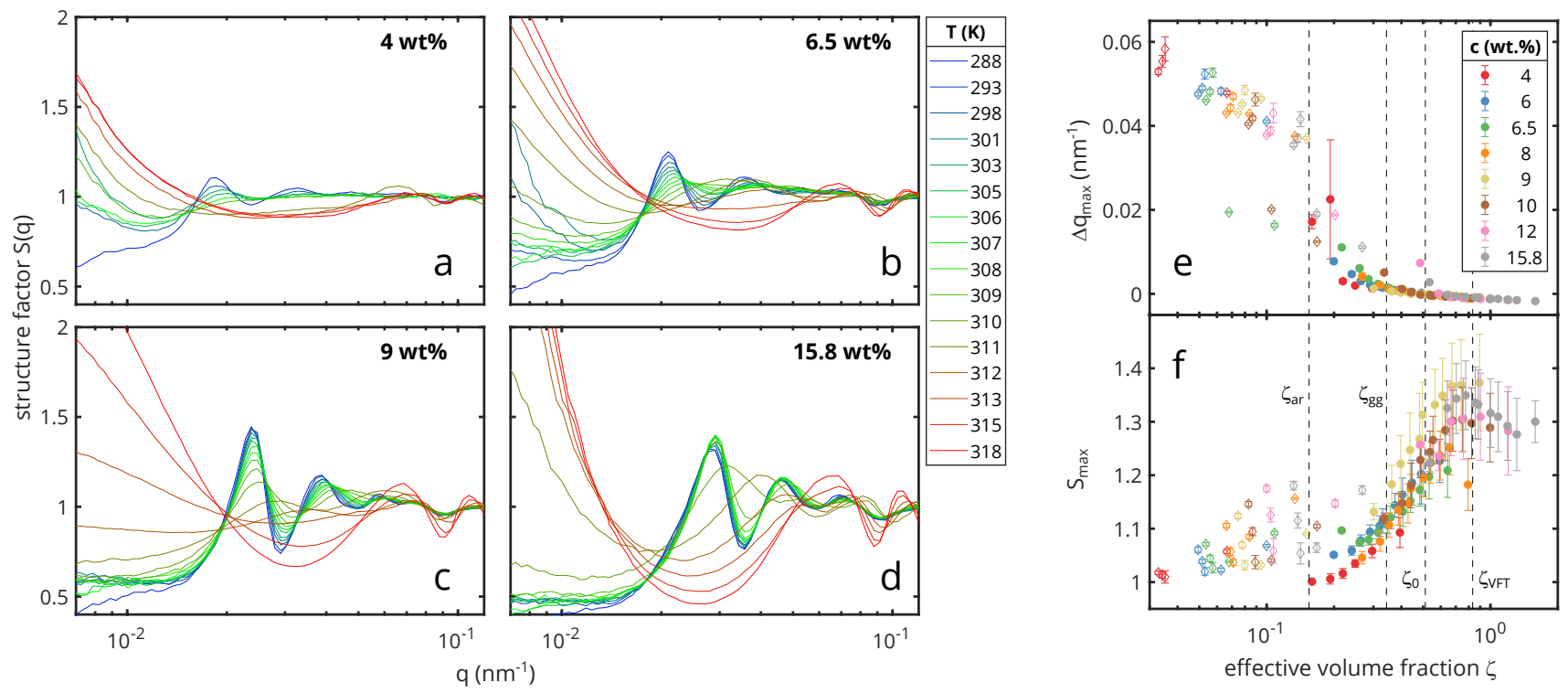

FIG. 2. a)-d) Structure factors for selected weight fractions: a) 4 wt.\%, b) 6.5 wt.\%, c) 9 wt.\%, d) 15.8 wt.\%. e) Relative position $\Delta q_{\max }$ and f) height $S_{\max }=S\left(q_{\max }\right)$ of the structure factor peak for all studied samples.

at $q \rightarrow 0$, especially for low weight fractions. In contrast, $S(q)$ for the sample at $c=15.8$ wt.\% shows only weak modifications up to $310 \mathrm{~K}$, underlining the glass state at this weight fraction. Increasing the temperature above $310 \mathrm{~K}$ the structure factors show significant changes, in particular a strong increase at $q \rightarrow 0$ and thus a shift of $q_{\max }$. The overall similarities of $S(q)$ at all concentrations prove the transition from repulsive interactions at low temperatures towards attractive interactions above the gelation temperature $T_{\text {gel }}=310$ $\mathrm{K}$ as suggested previously for such core-shell systems [23, 26].

The values of $q_{\max }$ and $S_{\max }$ are shown in Fig. 2 (e) and (f) as a function of $\zeta$. In fact, $q_{\max }$ depends on the weight fraction and thus shows slightly different values at similar volume fractions (see Supplementary Material). In order to compare the different weight fractions, we show difference values $\Delta q_{\max }$ with respect to $q_{\max }\left(\zeta_{0}\right)$ at the respective weight fraction. These have been obtained by a fit to a power law at high volume fractions (see Supplementary Material). For $\zeta \gtrsim 0.15 q_{\max }$ slightly drops as discussed above. With decreasing volume fraction, $q_{\max }$ suddenly increases for $\zeta<\zeta_{\mathrm{ar}} \approx 0.155$ which marks the change from repulsive to attractive interaction at $T_{\text {gel }}$. Below $\zeta_{\text {ar }} q_{\max }$ increases linearly with decreasing $\zeta$. A similar behaviour can be found for $S_{\max }$ where $\zeta_{\text {ar }}$ divides as well two regimes. Note that $\zeta_{\text {ar }}$ is not a fixed volume fraction such as $\zeta_{0}$ or $\zeta_{\mathrm{VFT}}$ but rather divides the measured samples in 
an attractive and a repulsive regime which is driven by crossing the LCST. For $\zeta<\zeta_{\text {ar }}$ the peak height $S_{\max }$ is dominated by its temperature dependence, with a minimum at $T=313$ K (see Supplementary Material). Above $\zeta_{\text {ar }}$ the maximum $S_{\max }$ follows a master curve for all c. In particular, it increases with $\zeta$ up to a maximum at $\zeta_{\mathrm{vFT}}$ and drops slightly for larger $\zeta$. The decrease of $\Delta q_{\max }$ in combination with the increasing $S_{\max }$ above $\zeta_{\text {ar }}$ indicates increasing order accompanied with a densification, i.e., reduction of next-neighbour distances which is typical for colloidal liquids approaching crystallisation or vitrification. The maximum at $\zeta_{\text {VFT }}$ has also been reported for other soft colloids at the glass transition volume fraction and results from a competition between energy and entropy [12, 28, 30].

Our results indicate glass-liquid and glass-gel transitions in a soft colloidal core-shell system. Changing the temperature between $288 \mathrm{~K}$ and $318 \mathrm{~K}$, the PNIPAm shell expels water leading to a decreased particle size and thus change of the effective volume fraction $\zeta$. Despite a change of the interaction potential with temperature [15], we observe phase changes as function of effective volume fraction independent from temperature.

For weight fractions below $10 \%$ the relaxation times obtained by XPCS fall on a master curve showing a slowing down following a VFT type. Above $\zeta_{0}=0.51$ the samples fall out of equlibrium, forming a glass state expressed by a change of the $\zeta$-dependence of $\tau$ to a less steep, linear slowdown. The VFT volume fraction $\zeta_{\mathrm{VFT}}=0.84$ and the low kinetic fragility index $m^{*} \approx 1$ match results obtained from pure PNIPAm colloids measured at fixed temperature with different number densities [28, 30, 44]. Most importantly, the liquidglass transition shows universal features of soft particles, and only depends on the volume fraction of the PNIPAm particles. Especially in the liquid state, regions of both attractive interaction at small $\zeta$ and repulsive states with increasing $\zeta$ fall on the VFT master curve. Moreover, this VFT behaviour is independent from the particles' softness which changes with temperature in our study challenging the role of softness on the glass transition in such soft colloids [28, 29, 39, 40].

The dynamics of the two highest weight fractions studied follow the linear slowdown above $\zeta_{0}$. However, different from the samples at $c<10 \mathrm{wt} \%$ the relaxation time slows down again with decreasing $\zeta$ in the regime of attractive interactions. Together with the exponents $p \approx 1$ and $\gamma \approx 1.8$ in this regime, the results suggest a glass-gel transition around $\zeta_{\mathrm{gg}}=0.34$. The sample at $c=10 \mathrm{wt} . \%$ shows an intermediate behaviour, i.e., the dynamics follows the linear increase of $\tau$ above $\zeta_{0}$, but matches the VFT curve only for repulsive states. 
In the attractive regime, $\tau$ is approximately one order of magnitude slower than for the liquid samples. Furthermore, the exponents suggest heterogeneous, subdiffusive dynamics. Interestingly, at $288 \mathrm{~K}$ this sample's volume fraction is $\zeta(10 \%, 288 \mathrm{~K})=1.0$. Below $\zeta=1$, the particles deform and their effective radius was reported to be stable whereas for $\zeta>1$ interpenetration is observed $[11,47,49,50]$. The sample at $c=10 \mathrm{wt} . \%$ is at the border between both states. This indicates that once interpenetration of PNIPAm shells is present in the repulsive regime, i.e. $\zeta>1$, a glass-gel transition takes place, while otherwise the glass-liquid transition occurs.

A particular characteristic is observed at $\zeta_{\mathrm{gg}}$. As discussed in the previous paragraph, this volume fraction marks the transition between the glass and the gel state for $c>10$ wt.\%. On the other hand, it coincides with the maximum of $p$ and the minimum of $\gamma$, respectively. Such extrema have been reported for soft colloids close to their glass transition $[28,44]$ and have been related to stress relaxation. In those studies, the sudden increase of $\gamma$ (and decrease of $p$ ) reflected a transition from stretched $(\gamma<1)$ to compressed $(\gamma>1)$ relaxation. This is different in our case, where $\gamma<1$ is found at both sides of the minimum. Instead, a sudden increase to a compressed exponential takes place between $\zeta_{0}$ and $\zeta_{\text {vFT }}$ and reflects the glass transition. Together with the coincidence with the glass-gel transition at larger weight fractions this results suggests a manifestation (or reminder) of the glass-gel transition in the supercooled liquid state. This is rationalized by the interference of glass and gel dynamics that results in anomalous dynamics such as subdiffusion [55] and multiple relaxation processes [56-58]. Both may result in a reduction of $\gamma$ and increase of $p$ which matches our data.

Compared to the dynamics, the structure factors show a different behaviour. First, $S(q)$ is almost constant at large $\zeta$ (see Fig. 2). This suggests a similar structure at high volume fractions due to deformation, deswelling and interpenetration where the dynamics change linearly with $\zeta$. Moreover, relative changes of $S(q)$ follow master curves that reflect the attractive and repulsive regime. In contrast, absolute values of $q_{\max }$ and $S_{\max }$ are defined by weight fraction $c$ and $T$, resembling the changing interaction potential due to the swelling of the particles. Interestingly, the dynamics are independent from that change.

The results are summarized in the $T$ - $\zeta$ phase diagram shown in Fig. 3. It extends the phase diagram published recently [26]. The reported volume fractions that mark phase borders as well as transitions of structure and dynamics are indicated. In conclusion, our 


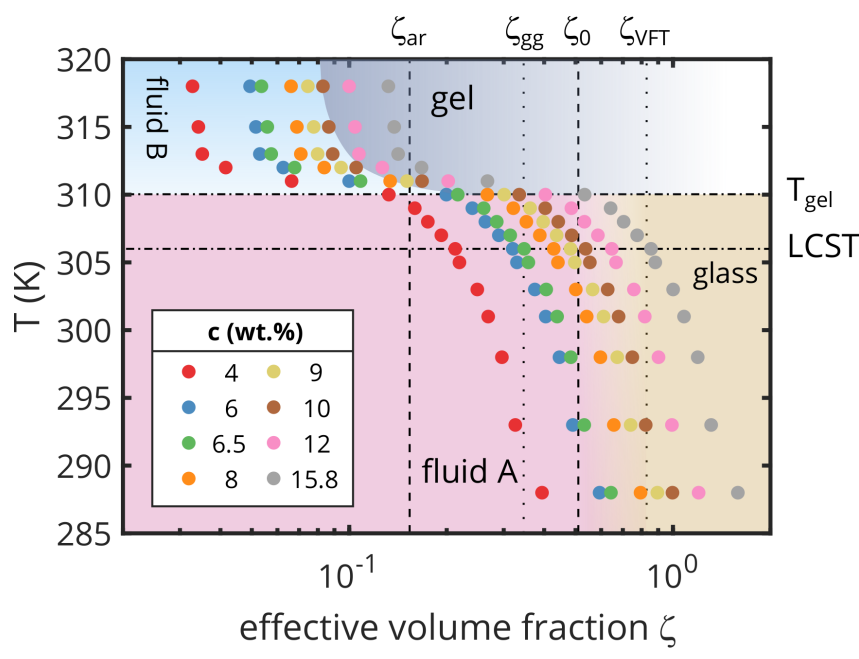

FIG. 3. T- $\zeta$ phase diagram for all samples studied. Colloidal gel regime adapted from [26]. Fluid A refers to the repulsive fluid, fluid B to the attractive fluid state. The glass state is found above $\zeta_{0}$

study on glass-gel and glass-liquid transitions of a thermo-responsive colloidal soft-sphere system provides a detailed view on their structure and dynamics over a wide area of the phase diagram. First, for $c<10$ wt.\% the volume fraction defines the phase, there is only a minor impact from the temperature and thus the interaction potential. Consequently, we found repulsive and attractive liquid phases whose dynamics fall on a joint master curve. Approaching the glass transition, the dynamics follow a VFT law with a kinetic fragility index of approximately 1. Second, if the particle shells are subject to deformation, deswelling as well as interpenetration at low temperatures ( $c>10$ wt.\%), a glass-gel transition is found at $\zeta_{\mathrm{gg}}$ upon heating. Third, we found subdiffusive dynamics in the liquid state with exponents $p, \gamma$ showing a maximum and minimum at $\zeta_{\mathrm{gg}}$ suggesting interference of the glassgel transition as a precursor in the liquid phase. These results motivate new studies in theory and experiments to fully unterstand the phase behaviour of soft particles at intermediate and high volume fractions.

We thank Francesco Dallari for discussion. This work is supported by the Cluster of Excellence "Advanced Imaging of Matter" (EXC 2056 - project ID 390715994) of the Deutsche Forschungsgemeinschaft (DFG). Parts of this research were carried out at the light source 
PETRA III at DESY, a member of the Helmholtz Association (HGF).

[12] J. Brijitta and P. Schurtenberger, Responsive hydrogel colloids: Structure, interactions, phase
behaviour, and equilibrium and non-equilibrium transitions of microgel dispersions, Curr.

[12] J. Brijitta and P. Schurtenberger, Responsive hydrogel colloids: Structure, interactions, phase
behaviour, and equilibrium and non-equilibrium transitions of microgel dispersions, Curr. 410, 259 (2001).

[2] L. Berthier and G. Biroli, Theoretical perspective on the glass transition and amorphous materials, Rev. Modern Phys. 83, 587 (2011).

[3] G. L. Hunter and E. R. Weeks, The physics of the colloidal glass transition, Rep. Prog. Phys. 75, 066501 (2012).

[4] P. N. Pusey and W. van Megen, Phase behaviour of concentrated suspensions of nearly hard colloidal spheres, Nature 320, 340 (1986).

[5] F. Sciortino and P. Tartaglia, Glassy colloidal systems, Adv. Phys. 54, 471 (2005).

[6] H. Tanaka, H. Tong, R. Shi, and J. Russo, Revealing key structural features hidden in liquids and glasses, Nat. Rev. Phys. 1, 333 (2019).

[7] F. Lehmkühler, B. Hankiewicz, M. A. Schroer, L. Müller, B. Ruta, D. Sheyfer, M. Sprung, K. Tono, T. Katayama, M. Yabashi, T. Ishikawa, C. Gutt, and G. Grübel, Slowing down of dynamics and orientational order preceding crystallization in hard-sphere systems, Sci. Adv. 6, eabc5916 (2020).

[8] E. B. Sirota, H. D. Ou-Yang, S. K. Sinha, P. M. Chaikin, J. D. Axe, and Y. Fujii, Complete phase diagram of a charged colloidal system: A synchro- tron x-ray scattering study, Phys. Rev. Lett. 62, 1524 (1989).

[9] M. Das, H. Zhang, and E. Kumacheva, MICROGELS: Old materials with new applications, Annu. Rev. Mater. Res. 36, 117 (2006).

[10] P. J. Yunker, K. Chen, M. D. Gratale, M. A. Lohr, T. Still, and A. G. Yodh, Physics in ordered and disordered colloidal matter composed of poly(n-isopropylacrylamide) microgel particles, Rep. Prog. Phys. 77, 056601 (2014).

[11] M. Karg, A. Pich, T. Hellweg, T. Hoare, L. A. Lyon, J. J. Crassous, D. Suzuki, R. A. Gumerov, S. Schneider, I. I. Potemkin, and W. Richtering, Nanogels and microgels: From model colloids to applications, recent developments, and future trends, Langmuir 35, 6231 (2019).

[1] P. G. Debenedetti and F. H. Stillinger, Supercooled liquids and the glass transition, Nature 
Opin. Colloid Interface Sci. 40, 87 (2019).

[13] F. Scheffold, Pathways and challenges towards a complete characterization of microgels, Nat. Commun. 11, 4315 (2020).

[14] P. S. Mohanty, D. Paloli, J. J. Crassous, E. Zaccarelli, and P. Schurtenberger, Effective interactions between soft-repulsive colloids: Experiments, theory, and simulations, J. Chem. Phys. 140, 094901 (2014).

[15] M. J. Bergman, N. Gnan, M. Obiols-Rabasa, J.-M. Meijer, L. Rovigatti, E. Zaccarelli, and P. Schurtenberger, A new look at effective interactions between microgel particles, Nat. Commun. 9, 5039 (2018).

[16] A. Zaccone, J. J. Crassous, B. Béri, and M. Ballauff, Quantifying the reversible association of thermosensitive nanoparticles, Phys. Rev. Lett. 107, 168303 (2011).

[17] S. L. Fussell, K. Bayliss, C. Coops, L. Matthews, W. Li, W. H. Briscoe, M. A. Faers, C. P. Royall, and J. S. van Duijneveldt, Reversible temperature-controlled gelation in mixtures of pNIPAM microgels and non-ionic polymer surfactant, Soft Matter 15, 8578 (2019).

[18] T. Kureha, H. Minato, D. Suzuki, K. Urayama, and M. Shibayama, Concentration dependence of the dynamics of microgel suspensions investigated by dynamic light scattering, Soft Matter 15, 5390 (2019).

[19] H. Wang, X. Wu, Z. Zhu, C. S. Liu, and Z. Zhang, Revisit to phase diagram of poly(nisopropylacrylamide) microgel suspensions by mechanical spectroscopy, J. Chem. Phys. 140, $024908(2014)$.

[20] J. Appel, N. de Lange, H. M. van der Kooij, T. van de Laar, J. B. ten Hove, T. E. Kodger, and J. Sprakel, Temperature controlled sequential gelation in composite microgel suspensions, Part. Part. Syst. Char. 32, 764 (2015).

[21] J. Appel, B. Fölker, and J. Sprakel, Mechanics at the glass-to-gel transition of thermoresponsive microgel suspensions, Soft Matter 12, 2515 (2016).

[22] T. Wang, L. Jin, Y. Song, J. Li, Y. Gao, and S. Shi, Rheological study on the thermoinduced gelation behavior of poly(n -isopropylacrylamide-co -acrylic acid) microgel suspensions, J. Appl. Polym. Sci. 134, 45259 (2017).

[23] L. Frenzel, F. Lehmkühler, I. Lokteva, S. Narayanan, M. Sprung, and G. Grübel, Anomalous dynamics of concentrated silica-PNIPAm nanogels, J. Phys. Chem. Lett. 10, 5231 (2019).

[24] A. Town, E. Niezabitowska, J. Kavanagh, M. Barrow, V. R. Kearns, E. García-Tuñón, and 
T. O. McDonald, Understanding the phase and morphological behavior of dispersions of syn-

[35] A. Basu, Y. Xu, T. Still, P. E. Arratia, Z. Zhang, K. N. Nordstrom, J. M. Rieser, J. P. Gollub, D. J. Durian, and A. G. Yodh, Rheology of soft colloids across the onset of rigidity: scaling behavior, thermal, and non-thermal responses, Soft Matter 10, 3027 (2014). 
[36] C. Pellet and M. Cloitre, The glass and jamming transitions of soft polyelectrolyte microgel suspensions, Soft Matter 12, 3710 (2016).

[37] S. K. Behera, D. Saha, P. Gadige, and R. Bandyopadhyay, Effects of polydispersity on the glass transition dynamics of aqueous suspensions of soft spherical colloidal particles, Phys. Rev. Materials 1, 055603 (2017).

[38] Q. Li, X. Peng, and G. B. McKenna, Long-term aging behaviors in a model soft colloidal system, Soft Matter 13, 1396 (2017).

[39] P. van der Scheer, T. van de Laar, J. van der Gucht, D. Vlassopoulos, and J. Sprakel, Fragility and strength in nanoparticle glasses, ACS Nano 11, 6755 (2017).

[40] V. Nigro, R. Angelini, M. Bertoldo, F. Bruni, M. A. Ricci, and B. Ruzicka, Dynamical behavior of microgels of interpenetrated polymer networks, Soft Matter 13, 5185 (2017).

[41] R. Rivas-Barbosa, E. Lázaro-Lázaro, P. Mendoza-Méndez, T. Still, V. Piazza, P. E. RamírezGonzález, M. Medina-Noyola, and M. Laurati, Different routes into the glass state for soft thermo-sensitive colloids, Soft Matter 14, 5008 (2018).

[42] S. Ciarella, R. A. Biezemans, and L. M. C. Janssen, Understanding, predicting, and tuning the fragility of vitrimeric polymers, Proceedings of the National Academy of Sciences 116, $25013(2019)$.

[43] A. Scotti, A. R. Denton, M. Brugnoni, J. E. Houston, R. Schweins, I. I. Potemkin, and W. Richtering, Deswelling of microgels in crowded suspensions depends on cross-link density and architecture, Macromolecules 52, 3995 (2019).

[44] V. Nigro, B. Ruzicka, B. Ruta, F. Zontone, M. Bertoldo, E. Buratti, and R. Angelini, Relaxation dynamics, softness, and fragility of microgels with interpenetrated polymer networks, Macromolecules 53, 1596 (2020).

[45] I. B. de Aguiar, T. van de Laar, M. Meireles, A. Bouchoux, J. Sprakel, and K. Schroën, Deswelling and deformation of microgels in concentrated packings, Sci. Rep. 7, 10223 (2017).

[46] A. Scotti, M. Pelaez-Fernandez, U. Gasser, and A. Fernandez-Nieves, Osmotic pressure of suspensions comprised of charged microgels, Phys. Rev. E 103, 012609 (2021).

[47] U. Gasser, J. S. Hyatt, J.-J. Lietor-Santos, E. S. Herman, L. A. Lyon, and A. FernandezNieves, Form factor of pNIPAM microgels in overpacked states, J. Chem. Phys. 141, 034901 (2014).

[48] P. S. Mohanty, S. Nöjd, K. van Gruijthuijsen, J. J. Crassous, M. Obiols-Rabasa, R. Schweins, 
A. Stradner, and P. Schurtenberger, Interpenetration of polymeric microgels at ultrahigh densities, Sci. Rep. 7, 1487 (2017).

[49] G. M. Conley, P. Aebischer, S. Nöjd, P. Schurtenberger, and F. Scheffold, Jamming and overpacking fuzzy microgels: Deformation, interpenetration, and compression, Sci. Adv. 3, e1700969 (2017).

[50] G. M. Conley, C. Zhang, P. Aebischer, J. L. Harden, and F. Scheffold, Relationship between rheology and structure of interpenetrating, deforming and compressing microgels, Nat. Commun. 10, 2436 (2019).

[51] A. Scotti, Characterization of the volume fraction of soft deformable microgels by means of small-angle neutron scattering with contrast variation, Soft Matter 17, 5548 (2021).

[52] C. A. Angell, Formation of glasses from liquids and biopolymers, Science 267, 1924 (1995).

[53] A. Scotti, M. Brugnoni, C. G. Lopez, S. Bochenek, J. J. Crassous, and W. Richtering, Flow properties reveal the particle-to-polymer transition of ultra-low crosslinked microgels, Soft Matter 16, 668 (2020).

[54] H. Shamana, M. Grossutti, E. Papp-Szabo, C. Miki, and J. R. Dutcher, Unusual polysaccharide rheology of aqueous dispersions of soft phytoglycogen nanoparticles, Soft Matter 14, 6496 (2018).

[55] E. Zaccarelli, I. Saika-Voivod, S. V. Buldyrev, A. J. Moreno, P. Tartaglia, and F. Sciortino, Gel to glass transition in simulation of a valence-limited colloidal system, J. Chem. Phys. 124, $124908(2006)$.

[56] P. Chaudhuri, L. Berthier, P. I. Hurtado, and W. Kob, When gel and glass meet: A mechanism for multistep relaxation, Physical Review E 81, 040502 (2010).

[57] P. Chaudhuri, P. I. Hurtado, L. Berthier, and W. Kob, Relaxation dynamics in a transient network fluid with competing gel and glass phases, J. Chem. Phys. 142, 174503 (2015).

[58] J. M. Olais-Govea, L. López-Flores, J. B. Zepeda-López, and M. Medina-Noyola, Interference between the glass, gel, and gas-liquid transitions, Sci. Rep. 9, 16445 (2019). 